\title{
Pharmaceutical Analytics at the Institute of Pharmaceutical Sciences
}

\author{
Hansruedi Altorfer*
}

\begin{abstract}
Some of our research activities in Pharmaceutical Analysis, Irradiation of Pharmaceutical Drug Substances, Development of Monographs and new Methods on Pharmaceutical Substances for the European- and Swiss Pharmacopoeia are described.
\end{abstract}

Keywords: Analytical methods · Ionizing radiation · Pharmaceuticals · Pharmacopoeia · Stability

In our view pharmaceutical analytics means how to obtain, to treat, and to interpret analytical data on drugs and drug-systems in order to develop new analytical strategies to reach more precise and accurate information concerning the 'true,' state of the investigated system. The results from our analytical investigations have a direct and important influence on the safety of drug-systems in human medicine. They may protect the patient from undesirable side-effects induced by the application of unqualified drugs. Our research activities have a worldwide effect because most of the analytical investigations are carried out in collaboration with international authorities (European Pharmacopoeia Commission, IAEA and WHO) and with different international pharmaceutical companies.

Two main fields of interest of our group are discussed in detail:

${ }^{*}$ Correspondence: Prof. Dr. H.R. Altorfer Institute of Pharmaceutical Sciences

ETH Hönggerberg

$\mathrm{CH}-8093$ Zürich

Tel.: +4116337351

Fax: +4116331315

E-Mail: altorfer@pharma.ethz.ch
The Investigation of the Effect of Ionizing Radiation ( $\gamma$-Rays and 10 MeV Electron beam) on Pharmaceutical Drug Substances and Drug-products

In line with our research collaborations with different pharmaceutical companies we are involved in many problems that can be easily formulated but are hard to solve analytically. The most frequently encountered question of a manufacturer is as simple as follows: Is it possible to perform $\gamma$ sterilization of a drug or its application form with an energy dose of 25 to $50 \mathrm{kGy}$ without causing any fundamental change of their specifications? The manufacturer expects the answer YES or NO, with a scientific proof of the reasons leading to the decision.

The analytical problems arising from $\gamma$ irradiation of drugs with high energy doses such as $25 \mathrm{kGy}$ are very complex and often unusual, since by-products might have been formed that are very different from those derived from conventional degradation mechanisms. Theoretical considerations are helpful, but in all cases a lot of experimental work has to be carried out [1-5]. Therefore, the analytical strategy for testing irradiated compounds with respect to their content and impurity profile is as follows: Many different analytical methods have to be screened in order to be sure that no radiolytic by-product has escaped detection. The pharmacopoeias of the different countries, although indicating valuable starting points for the analysis must be extended by additional tests. Furthermore, it has to be taken into account that the non-irradiated substance itself is 'impure' and its known composition is therefore taken as quasi- standard and compared with the irradiated substances. This procedure leads to different decision situations.

If one or more applied methods indicate a significant difference between the non-irradiated and the irradiated compound regarding the content and/or the impurity profile, the substance is classified to be not stable to $\gamma$-irradiation. Due to the amount of radiolytic by-products in general the following decision levels are reached:

1. If the sum of all radiolytic by-products amounts to more than 5\%, the substance is not worth further analytical investigations. The answer concerning the possibility of a successful $\gamma$-sterilization is definitely NO. No doubt is possible in this case.

2. If the sum of all radiolytic by-products is within the range of 0.5 to $2 \%$ the structure of the major radiolytic products should be elucidated and the amount of each should be estimated by at least two independent methods in order to prove accuracy and precision. If the generated radiolytic products are toxic and the irradiated substance could have harmful effects, the decision depends on toxicity measurements whether the substance is to be used for human or veterinary therapy or not. At the same time a likely pathway for the radiation-induced decomposition of the drug substance has to be stated, considering not only the pure compound itself but also the influence of the generally present impurities (by-products from the synthesis, degradation-products and, very important, the residual solvents) (Scheme). The knowledge of the irradiation induced decomposition path- 


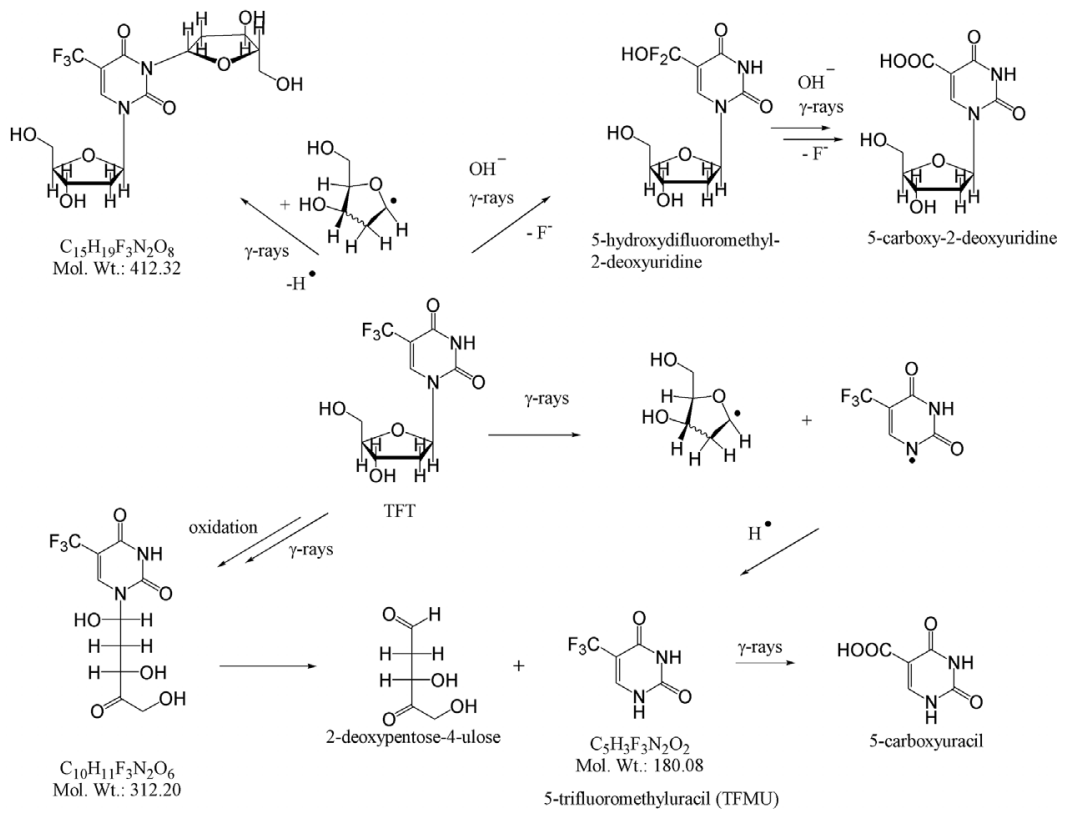

Scheme. Radiolytic decomposition pattern of trifluorothymidine
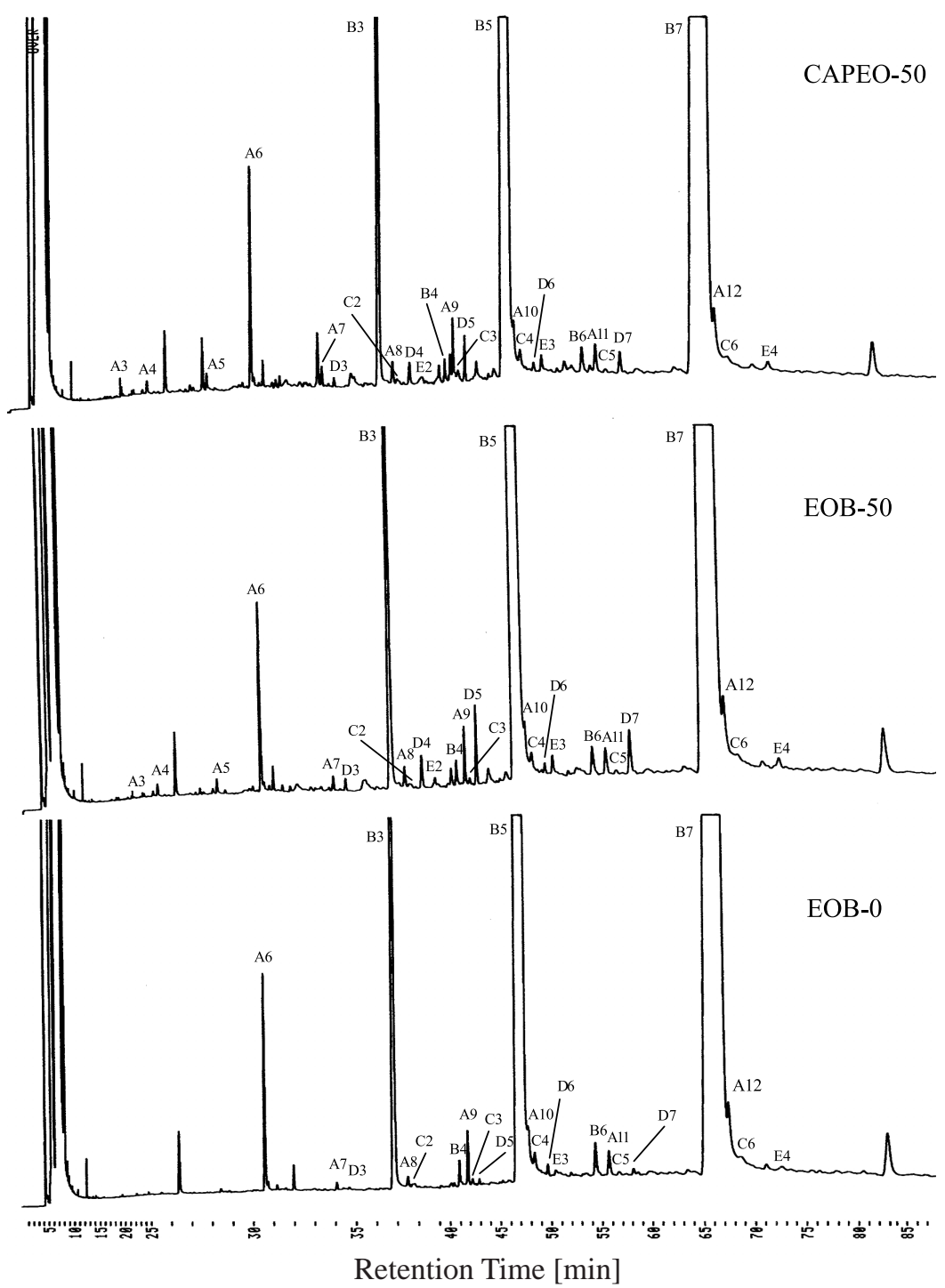

Fig. 1. Impurity chromatogram profiles of irradiated chloramphenicol eye ointment (CAPEO-50) and eye ointment base (EOB-50) as well as non-irradiated eye ointment (EOB-0), using a Rtx-5 ${ }^{\circledR}$ column. way can lead to modified radiation conditions, e.g. irradiation at lower temperature, change to inert-gas conditions or the use of radical scavengers. If the amount of the radiolytic by-products could be reduced to an acceptable level (Pharmacopoeia/1CHguidelines) by the modifications mentioned above, the answer concerning a successful $\gamma$-sterilization will be YES (Fig. 1). After this decision has been made, the analytical validation procedure requires a protocol for quality control along with its well-established rules to create a general prescription for characterization of the irradiated drug.

If none of the applied methods shows a significant difference between the irradiated and non-irradiated substance, and this is very important- the applied methods are different and relevant to the analytical problem, the answer concerning the possibility of $\gamma$-sterilization is YES.

With respect to liquid drug formulations, unfortunately in practice a positive decision is quite rare. The low energy required for fission of bonds in organic compounds is rather small compared to the applied irradiation energy and therefore the radiation stability of liquid systems is also theoretically less probable. In addition, secondary reactions induced by radiolytic products of the solvent(s) with the active ingredient are dominant and cannot be avoided. For almost dry and solvent-free drug substances there is a real chance to apply ionizing radiation as an alternative sterilization method, especially for thermo labile drugs.

At the present time six drug substances are under study at different stages.

\section{The Development of Monographs and New Methods on Pharmaceuti- cal Substances for the European and the Swiss Pharmacopoeia}

A monograph on a chemical substance is divided in six different sections:

- DEFINITION

- CHARACTERS

- PRODUCTION

- IDENTIFICATION

- TESTS

- ASSAY

Prior to the preparation of any monograph it is essential to accumulate as much information as possible on the substance in question. In particular it is necessary to ascertain:

- whether the substance is of natural, synthetic, semi-synthetic or biosynthetic origin;

- whether the substance is a mixture or a single entity;

- whether the method(s) of preparation are known in detail (method(s) of synthesis, isolation and purification); 
- whether there are different polymorphic forms, since the properties of the substance may vary in accordance with this parameter;

- whether the substance is available in pure enantiomeric form or as a racemic mixture;

- whether there are different hydrates or solvates;

- whether there are different salts.

The substance has to be defined with the greatest possible accuracy and precision with respect to the above-mentioned properties. Some chemical substances, particularly those obtained from raw materials of natural origin and substances produced by fermentation may not be easily separated from certain related substances (e.g. quinine salts, amino acids). Hence pharmaceuticals can be categorized as:

- a chemical product when obtained in a very pure state when they can be assayed by a physico-chemical method;

- a substance accompanied by a certain proportion of related substances, given an exact definition of the main component only (e.g. neomycin);

- a mixture of several components, sometimes difficult to define, where an overall description may suffice (e.g. nystatin).

The most important step during the elaboration of a monograph represents the choice of the analytical procedure (methods) for identification, purity testing and the assay of a drug substance [6-8].

The most complex and from the analytical point of view the most demanding part of a monograph represents the test section. In designing the purity tests for a monograph on a given material, interest is to be focused on limiting tests for those impurities that could possibly arise during the manufacture process, its degradation upon storage, or in case of biological materials, due to adulteration. Whenever possible, products of different origin are to be examined in this respect. Special attention must be paid to impurities that are known to possess or are suspected to have toxic properties or that could appreciably modify the activity and/or stability of the drug substance itself or of other materials with which it is usually associated. It must be recognized however, that not all impurities can be limited by means of general methods and specific tests and that a certain lack of specificity may even be desirable in order to provide some safeguard against the presence of unforeseen foreign substances in a material.

By-products (related substances) are known impurities which may be identified or unidentified. They include intermediates and products from a synthetically produced organic substance, co-extracted substances from a natural product, and degradation products of the substance. This definition does not include other possible impurities

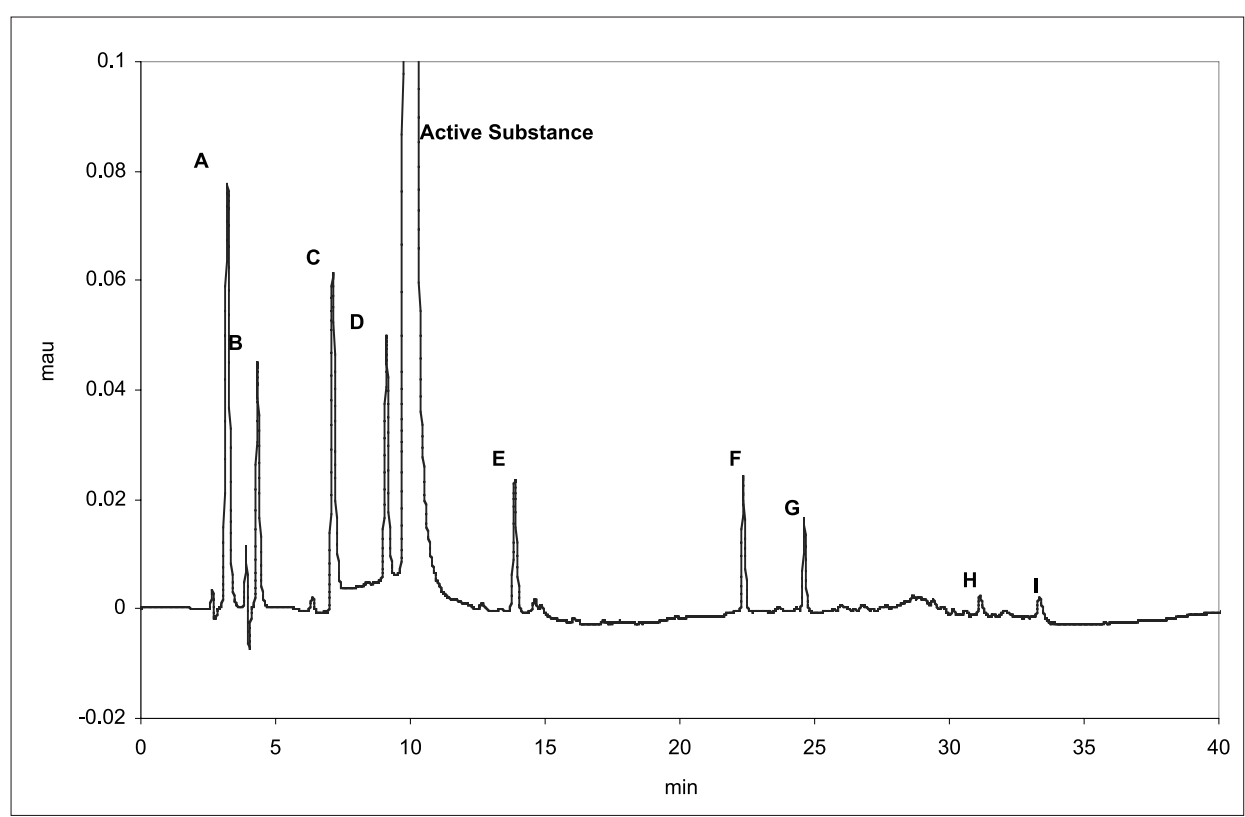

Fig. 2. HPLC impurity profile of a new drug substance (A-G: known-, $\mathrm{H}$ and I: unknown Impurities)

such as residual solvents, residues from cells and microorganisms or culture media used in fermentation processes. Related substances can be controlled by various spectroscopic or chromatographic methods or combinations of these.

Whichever approach is taken to control the impurities in a drug-substance, knowledge of the synthetic route and the likely decomposition pathways is essential to develop a test for related substances that is highly discriminatory and that can separate the suspected impurities from the substance itself (Fig. 2). In case of different methods of synthesis used by various manufacturers, the analytical techniques described should check for the major impurities from all syntheses. Detection limits must be established for impurities of known structure. The discrimination power of an analytical impurity test, its repeatability, its reproducibility and accuracy must be determined by means of collaborative studies (validation). The limits set for the control of impurities will depend on a number of factors including toxicity, route of administration and the duration of treatment of the drug substance. In newly developed drugsubstances, unidentified impurities normally are not allowed to exceed the level of $0.1 \%$.

At the moment monographs on three new substances for the European Pharmacopoeia and one substance for the Swiss Pharmacopoeia are under study in our group. During our research activities we have developed some new analytical methods in collaboration with industries and pharmacopoeia authorities [9-12].

Received: July 13, 2004

[1] I.A. Werner, H.R. Altorfer, 'The Importance of Analytical Chemistry for Irradiated Drugs', beta/gamma 1990, 3(4), 25.
[2] H.R. Altorfer, Proceedings of the $9^{\text {th }}$ International Symposium on Instrumental Planar Chromatography, 1997, 3, 15-46.

[3] P. Horsch, L. Bigler, H.R. Altorfer, 'Influence of radiation sterilization on the stability of trifluorothymidine', Int. J. Pharma. 2001, 22, 205-215

[4] L. Hong, H.R. Altorfer, 'Determination of assay and impurities of gamma irradiated chloramphenicol in eye ointment', $J$. Pharm. Biomed. Anal. 2001, 24, 1-15.

[5] L. Hong, H.R. Altorfer, 'Characterization of gamma irradiated eye ointment base by headspace-gas chromatography-mass spectrometry', J. Pharm. Biomed. Anal. 2002, 29(1-2), 263-275.

[6] Pharmacopoeia Helvetica 2004, Ed. 9.

[7] The European Pharmacopoeia Convention, European Pharmakopoea 2004, Ed. 4.08.

[8] The United States Pharmacopoeia Convention Inc., 2004, USP 27.

[9] L. Hong, H.R. Altorfer, 'A microsized headspace $\mathrm{GC}$ technique for determination of organic volatile impurities in water insoluble pharmaceuticals', Chromatographia, 2000, 53, 76-80.

[10] C. Schatz, M. Ulmschneider, R. Altermatt, S. Marrer, H.R. Altorfer, 'Thoughts on Fiber Optics in Dissolution Testing', Dissolution Technologies, 2001, Vol. 8/2/May.

[11] H.R. Altorfer, L.A. Crosbie, J.H. Miller, 'An improved liquid chromatographic method for the control of Nitriloacetic Acid (NTA) in Edetic Acid and its salts for inclusion in the relevant monographs', PHARMEUROPA, 2002, Vol. 14, No. 4, October.

[12] S. Freimüller, H.R. Altorfer, "A chiral HPLC method for the determination of low amounts of D-Carnitine in L-Carnitine after derivatization with $(+)$ FLEC', $J$. Pharm. Biomed. Anal. 2002, 30(2), 202-218. 